\title{
Acceleration of FDTD radiation pattern calculation using two-dimensional ARMA
}

\author{
Akihiro Nakao $^{1}$, Takuji Arima ${ }^{1,}$ a), and Toru Uno ${ }^{1}$ \\ ${ }^{1}$ Graduate School of Engineering, Tokyo University of Agriculture and Technology \\ Naka-cho, Koganei-shi, Tokyo 184-8588, Japan
}

a)t-arima@cc.tuat.ac.jp

\begin{abstract}
In recent years, the finite-difference time-domain (FDTD) method has been widely employed for analyzing various electromagnetic problems, including low-frequency problems. It provides accurate results in most cases, but it requires relatively long calculation time for slow convergence problems, such as array antennas. For this reason, the autoregressive moving average (ARMA) model has been applied to the FDTD analysis to reduce calculation time. However, it has not been applied to FDTD radiation pattern analysis because the radiation patterns calculation requires multi-dimensional (time and space) data. In this letter, two-dimensional ARMA is applied to FDTD radiation pattern analysis to reduce the calculation time. We confirm effectiveness of the method by calculating the radiation pattern of Yagi-Uda antenna.
\end{abstract}

Keywords: FDTD method, ARMA algorithm, two-dimensional ARMA Classification: Antennas and Propagation

\section{References}

[1] T. Uno, Y. He, and T. Arima, FDTD Method for Computational Electromagnetics: Fundamentals and Practical Applications, Corona Pub., 2016 (in Japanese).

[2] K. Asano, T. Uno, and T. Arima, "Acceleration of FDTD calculation of EM fields due to loop antennas used for $\mathrm{MHz}$ band wireless transfer system placed near human body," IEICE Commun. Express, vol. 6, no. 6, pp. 325-330, 2017. DOI: 10.1587/comex.2016spl0036

[3] T. Sekiguchi, T. Hikage, M. Yamamoto, T. Nojima, S. Futatsumori, K. Morioka, A. Kohmura, and N. Yonemoto, "Numerical estimation of propagation path loss for wireless link design of WAIC systems installed on outside aircraft cabin based on large-scale FDTD simulation," IEICE Commun. Express, vol. 8, no. 5, pp. 129-134, 2019. DOI: 10.1587/comex.2018xb10165

[4] S. Futatsumori, K. Morioka, A. Kohmura, N. Sakamoto, T. Soga, and N. Yonemoto, "Feasibility evaluations of three-dimensional-printed high-gain reflectarray antenna for W-band applications," IEICE Commun. Express, vol. 7, no. 6, pp. 230-235, 2018. DOI: 10.1587/comex.2018xb10038

[5] T. Uno, "Electromagnetic modeling of metamaterials," IEICE Trans. Commun., vol. E96-B, no. 10, pp. 2340-2347, 2013. DOI: 10.1587/transcom.e96.b.2340

[6] J. Zielinski, N. Bouaynaya, and D. Schonfeld, "Two-dimensional ARMA modeling for breast cancer detection and classification,” Proc. IEEE Int. Conf Signal 


\section{Introduction}

The finite-difference time-domain (FDTD) method [1] is widely employed for analyzing various electromagnetic problems, such as low-frequency problems [2], propagation modeling [3], and radar modeling [4]. Simplicity is one of the strengths of the FDTD method. Moreover, the time step of the FDTD method should keep the Courant limit [1]; therefore, it requires a lot of iterations to analyze slow conversion problems like array antennas. To reduce calculation time, prediction methods have been applied to the FDTD method. One of the prediction methods is using the autoregressive moving average (ARMA) model [5] to predict convergence values. It can reduce the calculation time of the FDTD analysis; the effectiveness of the ARMA model in the FDTD analysis has been verified [2, 5]. However, the ARMA model has not been applied to FDTD radiation pattern analysis because the radiation patterns calculation requires multi-dimensional (time and space) data. In this letter, two-dimensional ARMA [6] is applied to FDTD radiation pattern analysis to reduce the calculation time.

\section{Two-dimensional ARMA for FDTD radiation pattern analysis}

In this section, we explain the two-dimensional ARMA model for FDTD radiation pattern analysis. In the FDTD radiation pattern analysis, we use time-domain equivalent currents, $\boldsymbol{M}_{\boldsymbol{S}}=\boldsymbol{E} \times \hat{n}$ and $\boldsymbol{J}_{\boldsymbol{s}}=\hat{n} \times \boldsymbol{H}$, on the closed surface to calculate time-domain directional function, $\boldsymbol{D}$. In this letter, we consider calculating a onecut-plane radiation pattern (Fig. 1). To calculate the one-cut-plane radiation pattern, time-domain directional function $\boldsymbol{D}(\theta, t)$ given by:

$$
\left.\begin{array}{c}
D_{\theta}(\theta, t)=-Z_{0} \frac{W_{\theta}(\theta, t)}{d t}-\frac{U_{\emptyset}(\theta, t)}{d t} \\
D_{\emptyset}(\theta, t)=-Z_{0} \frac{d W_{\emptyset}(\theta, t)}{d t}+\frac{d U_{\theta}(\theta, t)}{d t}
\end{array}\right\}
$$

where $\boldsymbol{W}$ and $\boldsymbol{U}$ are given by:

$$
\left.\begin{array}{l}
\boldsymbol{W}(\theta, t)=\frac{1}{4 \pi c} \frac{\partial}{\partial t}\left\{\oint_{S} \boldsymbol{J}_{\boldsymbol{S}}\left(t+\frac{\hat{r} \cdot \boldsymbol{r}^{\prime}}{c}\right) d S^{\prime}\right\} \\
\boldsymbol{U}(\theta, t)=\frac{1}{4 \pi c} \frac{\partial}{\partial t}\left\{\oint_{S} \boldsymbol{M}_{\boldsymbol{S}}\left(t+\frac{\hat{r} \cdot \boldsymbol{r}^{\prime}}{c}\right) d S^{\prime}\right\}
\end{array}\right\} .
$$

The $\boldsymbol{D}$ is a function of both time $t$ and angle $\theta$. In the ARMA model, the input signals $x(\theta, z)$ and output signals $y(\theta, z)$ are used, where $z$ is a parameter of the $z$-transformation, $z^{n}=\exp \left(j \omega T_{n}\right), T_{n}$ is a sampling time in the FDTD method.

In the FDTD analysis, $x(\theta, z)$ corresponds to the excitation voltage of the antenna, and $y(\theta, z)$ corresponds to the time-domain directional function $\boldsymbol{D}(\theta, t)$. The relationship between the input and output signals is as follows:

$$
B_{0} y(\theta, z)=-\sum_{i=1}^{p 1} B_{i} y(\theta-i, z)+\sum_{j=0}^{q 1} A_{j} x(\theta-j, z)
$$




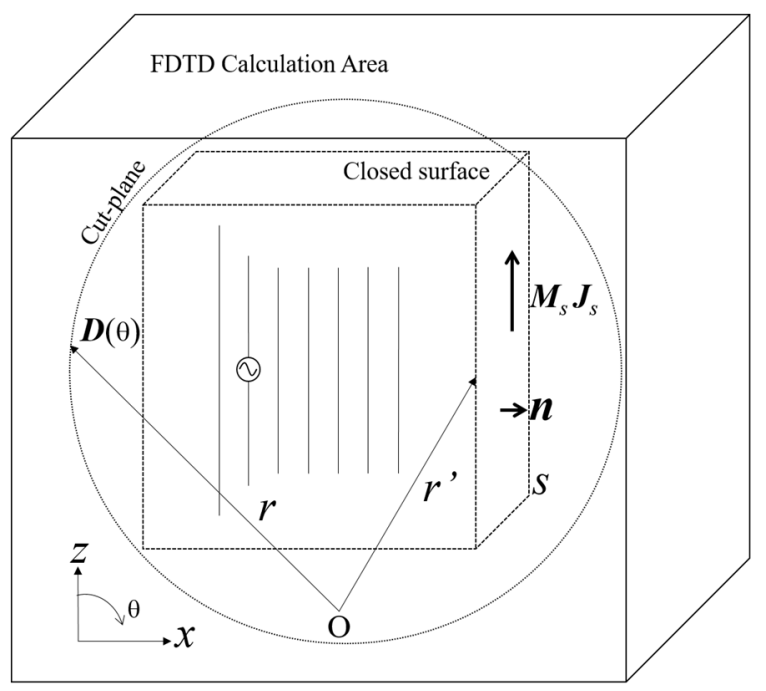

Fig. 1. FDTD radiation pattern analysis

where

$$
B_{i}=\sum_{k=0}^{p 2} b_{i k} z^{-k}, \quad A_{j}=\sum_{l=0}^{q 2} a_{j l} z^{-l} .
$$

$b_{i k}$ and $a_{j l}$ are the unknown coefficients to be determined. To obtain the unknown coefficients, Eq. (3) can be expressed in matrix form as follows:

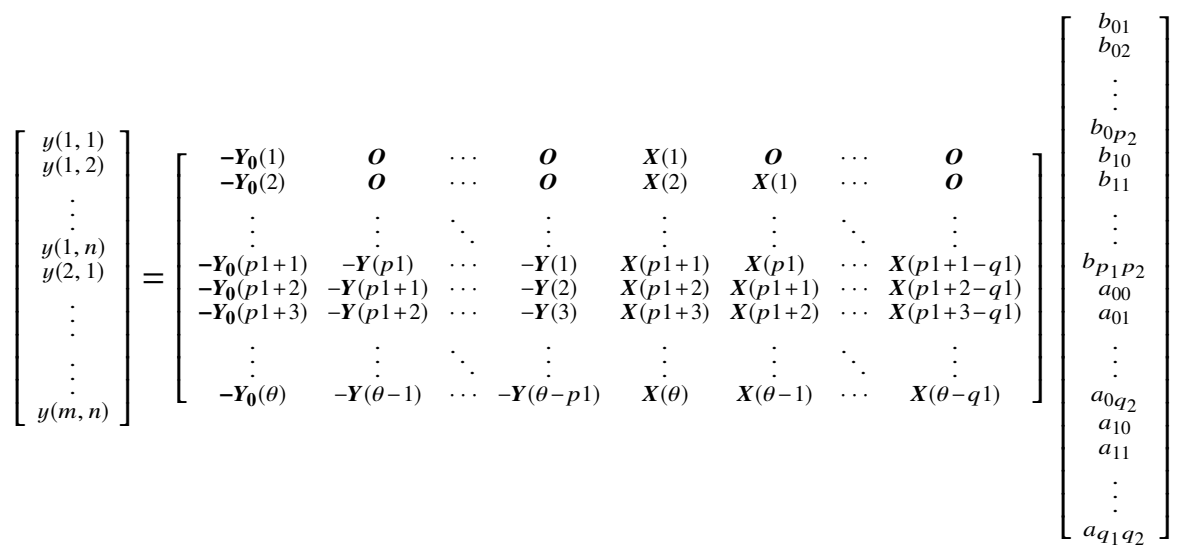

where

$$
\mathbf{Y}_{\mathbf{0}}(i)=\left[\begin{array}{cccc}
0 & 0 & \cdots & 0 \\
-y(i, 1) & 0 & \cdots & 0 \\
\vdots & \vdots & \ddots & \vdots \\
-y(i, p 2) & -y(i, p 2-1) & \cdots & -y(i, 1) \\
-y(i, p 2+1) & -y(i, p 2) & \cdots & -y(i, 2) \\
-y(i, p 2+2) & -y(i, p 2+1) & \cdots & -y(i, 3) \\
\vdots & \vdots & \ddots & \vdots \\
-y(i, n-1) & -y(i, n-2) & \cdots & -y(i, n-p 2)
\end{array}\right]
$$




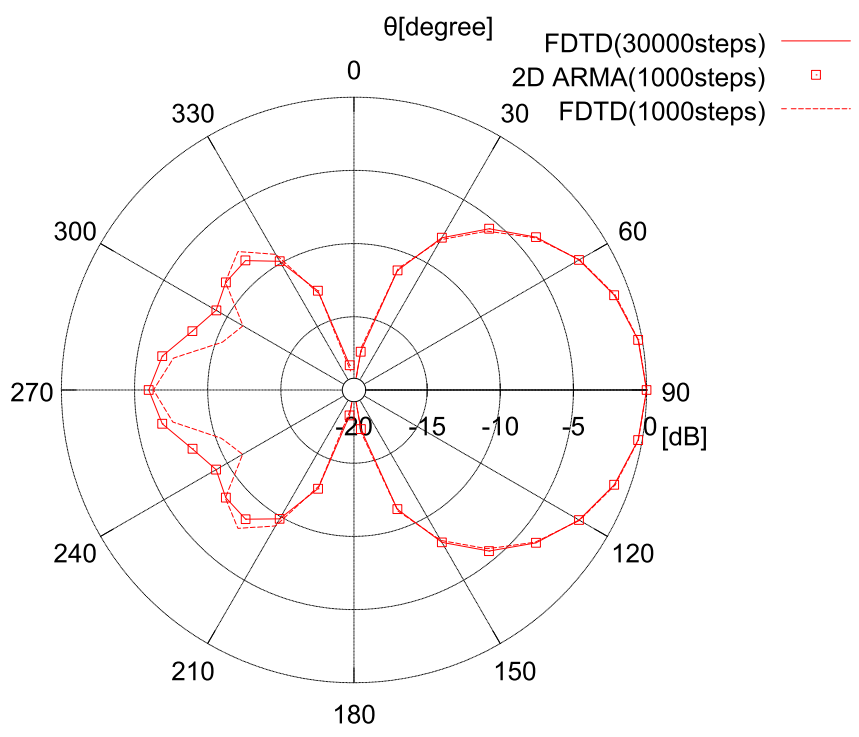

(a) Radiation pattern at $1.5 \mathrm{GHz}$

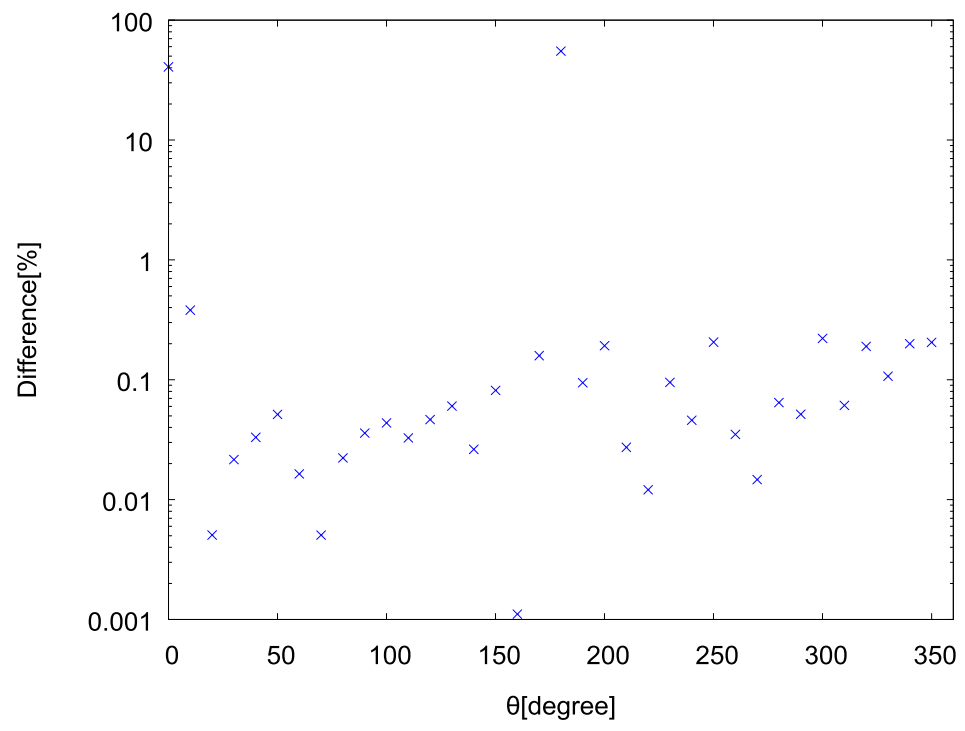

(b) The difference between traditional and adapted FDTD methods

Fig. 2. Calculation results

Table I. Calculation time

\begin{tabular}{l|c|c}
\hline & FDTD(30000steps) & 2D ARMA(1000steps+ARMA) \\
\hline Calculation time & $226 \mathrm{~m} 45 \mathrm{~s}$ & $16 \mathrm{~m} 34 \mathrm{~s}$ \\
\hline
\end{tabular}

$$
\mathbf{Y}(i)=\left[\begin{array}{cccc}
y(i, 1) & 0 & \cdots & 0 \\
y(i, 2) & y(i, 1) & \cdots & 0 \\
\vdots & \vdots & \ddots & \vdots \\
y(i, p 2+1) & y(i, p 2) & \cdots & y(i, 1) \\
y(i, p 2+2) & y(i, p 2+1) & \cdots & y(i, 2) \\
y(i, p 2+3) & y(i, p 2+2) & \cdots & y(i, 3) \\
\vdots & \vdots & \ddots & \vdots \\
y(i, n) & y(i, n-1) & \cdots & y(i, n-p 2)
\end{array}\right]
$$




$$
\mathbf{X}(i)=\left[\begin{array}{cccc}
x(i, 1) & 0 & \cdots & 0 \\
x(i, 2) & x(i, 1) & \cdots & 0 \\
\vdots & \vdots & \ddots & \vdots \\
x(i, p 2+1) & x(i, p 2) & \cdots & x(i, p 2+1-q 2) \\
x(i, p 2+2) & x(i, p 2+1) & \cdots & x(i, p 2+2-q 2) \\
x(i, p 2+3) & x(i, p 2+2) & \cdots & x(i, p 2+3-q 2) \\
\vdots & \vdots & \ddots & \vdots \\
x(i, n) & x(i, n-1) & \cdots & x(i, n-q 2)
\end{array}\right]
$$

Finally, the prediction value of the directional function $\boldsymbol{D}$ can be obtained by using Eq. (3), the coefficients $b_{i j}$ and $a_{i j}$ can be obtained by solving above matrix.

\section{Calculation results}

Fig. 1 shows the analyzed antenna and coordinate. This antenna is a 7 elements Yagi-Uda antenna. The length of the driven element, reflector element, and director element are 12, 10, and $8 \mathrm{~mm}$, respectively. The size of FDTD calculation area is $150 * 150 * 150$ cells include 8 -layer PML absorbing boundary. The cell size is $1 * 1 * 1$ cm. Fig. 2(a) shows the calculated radiation patterns. In the calculation, the number of time steps of the adapted method is 1000 steps. The values of the unknown coefficients $p_{1}, p_{2}, q_{1}$, and $q_{2}$ are set as 36, 95, 36, 95, respectively. The numbers of $p_{1}, p_{2}, q_{1}$, and $q_{2}$ are determined as large numbers for keeping high accuracy. In this calculation, 200 time steps data in total time steps (1000) data and 72 points angle data were used for the 2-D ARMA algorithm. In the angle calculation, the data of 360-720 degrees are set as zero. Therefore, $p_{1}$ and $q_{1}$ are half of the number of angle data, $p_{2}$ and $q_{2}$ are $40-50 \%$ for time step data. The calculated result of the adapted method is consistent with that of traditional FDTD method. Fig. 2(b) shows the difference between the traditional and the adapted FDTD methods. We observed a relatively large difference at 0 , and180 degrees; it is because 0 , and 180 degrees are the null angle of the antenna.

Table I shows the calculation time for each method. In this calculations, we used Linux server (CPU: Intel(R) Xeon(R) CPU E5-2640 v3 @ 2.60GHz). In the FDTD calculation, 643 Mbytes of memory is used. In 2D ARMA calculation, 2,058 Mbytes of memory is used. The calculation time of the adapted method is about $1 / 10$ or less compared with the traditional FDTD method.

\section{Conclusion}

In this letter, we applied two-dimensional ARMA to FDTD radiation pattern analysis. We realized that the calculation result of the adapted FDTD is consistent with that of the traditional FDTD. In addition, the number of time steps required for analysis reduced. Thus, two-dimensional ARMA is effective for FDTD radiation pattern analysis. 